\title{
Reflets
}

Revue ontaroise d'intervention sociale et communautaire

\section{Coalition des communautés en santé}

\section{Joanne Patoine}

Volume 1, numéro 1, printemps 1995

Des pratiques à notre image : défis et ressources

URI : https://id.erudit.org/iderudit/026064ar

DOI : https://doi.org/10.7202/026064ar

Aller au sommaire du numéro

Éditeur(s)

Reflets : Revue ontaroise d'intervention sociale et communautaire

ISSN

1203-4576 (imprimé)

1712-8498 (numérique)

Découvrir la revue

Citer cet article

Patoine, J. (1995). Coalition des communautés en santé. Reflets, 1(1), 207-212.

https://doi.org/10.7202/026064ar

Tous droits réservés (C) Reflets : Revue ontaroise d'intervention sociale et communautaire, 1995

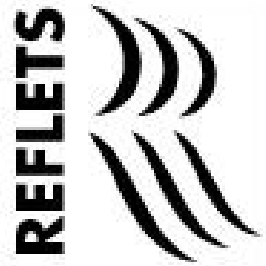

Ce document est protégé par la loi sur le droit d'auteur. L'utilisation des services d'Érudit (y compris la reproduction) est assujettie à sa politique d'utilisation que vous pouvez consulter en ligne.

https://apropos.erudit.org/fr/usagers/politique-dutilisation/ 


\section{Coalition des communautés en santé}

Joanne Patoine

Animatrice / Coordonnatrice pour les francophones de l'Ontario

Le concept des communautés en santé s'appuie sur une vision holistique préconisant l'utilisation d'approches globales pour favoriser l'amélioration de la santé des personnes et des collectivités, par le biais de politiques publiques saines et de projets de développement communautaire. La responsabilité des communautés en santé repose sur les collectivités locales; le mouvement veut encourager les gouvernements locaux à prendre des décisions et à établir des politiques qui privilégient la promotion de la santé, tout en suscitant la participation des citoyennes et des citoyens comme partenaires actifs de ce processus.

Après avoir situé le contexte historique du mouvement des Communautés en santé, je présenterai sa structure et ses modes de fonctionnement, pour ensuite discuter de son implantation en Ontario français.

\section{Le développement des communautés en santé}

Le mouvement des communautés en santé débute en 1974, suite à la publication, par le gouvernement fédéral, du rapport intitulé Nouvelle perspective de la santé des Canadiens, le rapport Lalonde (ministère de la Santé nationale et du Bien-être, 1974). Ce rapport élargissait la définition de la santé en identifiant ses principaux 
Reflets

déterminants comme étant la biologie humaine, l'environnement, le mode de vie et les organismes de soins de santé. Cette reconnaissance des liens étroits entre l'environnement social et la santé des individus amorce un mouvement qui cherche à aller au-delà du modèle médical traditionnel, vers une perspective plus globale de promotion de la santé.

Dix ans plus tard, une conférence internationale ayant pour thème Au-delà des soins de santé, a lieu à Toronto; son but est d'examiner les progrès accomplis depuis la publication du rapport Lalonde. Par la suite, l'Organisation mondiale de la santé (OMS) propose une définition de la santé comme étant la capacité d'une personne ou d'un groupe de réaliser ses aspirations et de satisfaire à ses besoins, de modifier l'environnement ou de pouvoir y faire face. La santé est donc perçue comme une ressource quotidienne, une dimension de la qualité de vie, et non comme l'objet de vie; il s'agit d'un concept positif qui met l'accent sur les ressources sociales et personnelles, de même que sur les capacités physiques de chaque individu (OMS,1986).

En 1986, la Charte d'Ottawa sur la promotion de la santé était promulguée sous les auspices de l'Organisation mondiale de la santé. Cette Charte définit la promotion de la santé comme étant «...le processus qui permet aux gens d'avoir un meilleur contrôle sur leur santé et de pouvoir l'améliorer... La promotion de la santé n'est pas la seule responsabilité du secteur de la santé, elle s'étend aussi à des modes de vie sains pour atteindre le mieuxêtre» (OMS, 1986).

À la suite de la publication de cette charte, le bureau européen de l'OMS lance le Projet des villes en santé. Engageant au départ dix villes, le mouvement des Communautés en santé a connu une croissance rapide et englobe aujourd'hui plus de mille municipalités et communautés participantes, et entre quinze et vingt réseaux nationaux ou régionaux florissants en Europe, en Amérique du Nord, en Australie, en Afrique occidentale et en Amérique latine.

Au Canada, c'est un projet conjoint de l'Institut canadien des urbanistes, de l'Association canadienne de santé publique et de la 
Fédération canadienne des municipalités qui a lancé le mouvement des Communautés en santé. Cette initiative reprend le modèle du projet européen des villes en santé et fait appel à la contribution d'organismes communautaires, d'urbanistes et de politiciennes et politiciens afin de favoriser une saine politique publique au palier municipal. Santé et Bien-être social Canada a subventionné le projet pendant trois ans. L'Institut canadien des urbanistes y contribue toujours en assumant une fonction de maillage pour des coalitions provinciales de Communautés en santé.

Ce sont les provinces de Québec et de Colombie-Britannique qui sont les plus actives au Canada et chacune reçoit des fonds de leur gouvernement provincial. En Colombie-Britannique, quatrevingt-onze communautés participantes profitent de l'aide d'un petit secrétariat qui facilite l'établissement de réseaux entre les membres, dispense des conseils, offre de la consultation, élabore des ressources pour diffuser l'information et favorise le développement de nouvelles compétences. Les soixante-quinze communautés participantes au Québec sont aussi appuyées par un petit secrétariat dont les responsabilités sont semblables à celles du secrétariat de la Colombie-Britannique. Le gouvernement de l'Ontario vient tout juste d'accorder des fonds et des ressources à la Coalition des communautés en santé de l'Ontario pour mettre en place un secrétariat ayant ces mêmes fonctions.

\section{Modalités et structure}

Deux processus parallèles ont été développés pour gouverner la Coalition des Communautés en santé: une assemblée générale annuelle (AGA) et un comité de direction. Les membres des groupes régionaux des Communautés en santé et les organisations provinciales participent à l'AGA. Chaque groupe et organisation provinciale a droit de vote.

Le comité de direction, composé de dix membres choisis par les six régions et de sept membres des organisations provinciales, 
Reflets

se rencontre régulièrement pour établir le plan stratégique de la Coalition. Les six régions, telles que déterminées par la Coalition, pourront être modifiées en fonction du nombre de groupes qui s'établiront dans les années à venir.

Les membres des comités tentent constamment d'assurer une représentation équitable de la population (ethnique, Premières Nations et francophone), soit par nomination, par élection et/ou par sélection.

Présentement, la Coalition est subventionnée par le ministère de la Santé et parrainée par le Centre ontarien d'information en prévention. Les associations qui y sont représentées sont les suivantes:

- Association des centres de santé de l'Ontario

- Conseil de la conservation de l'Ontario

- Association des architectes-paysagistes de l'Ontario

- Association médicale ontarienne

- Centre ontarien d'information en prévention

- Association pour la santé publique de l'Ontario

- Institut des planificateurs professionnels de l'Ontario

- Association des conseils régionaux de santé de l'Ontario

- Conseil du développement social de l'Ontario

- Société pour la gestion de l'environnement de l'Ontario

- L'Association des municipalités de l'Ontario participe comme membre de soutien.

\section{Les communautés en santé en Ontario français}

En tant qu'animatrice/coordinatrice pour les francophones de l'Ontario, mon rôle consiste, d'une part, à agir comme agente de liaison entre des personnes, des agences, des villes et parfois même avec d'autres provinces. Je peux, d'autre part, fournir des renseignements adaptés aux besoins des groupes qui proposent des initiatives, faciliter, avec les groupes intéressés, le développement de projets, ou encore aider à identifier les ressources disponibles. 
Présentement, la ville de Sudbury compte la seule initiative Communauté en santé reconnue comme étant exclusivement francophone. Il existe d'autres projets dans lesquels les francophones participent, notamment à Prescott-Russell. D'autres projets en voie de réalisation, sous l'auspice d'une municipalité ou du Conseil consultatif d'un maire, dans des régions avec une population francophone, possèdent tous les ingrédients nécessaires pour se rallier aux Communautés en santé.

L'initiative qui se développe à Sudbury tiendra lieu de projet pilote, orientant le bureau provincial dans ses efforts subséquents visant à encourager la mise en oeuvre d'initiatives spécifiques aux francophones de la province. Depuis la première assemblée générale annuelle qui a eu lieu à Port Elgin les 25 et 26 novembre 1994, des francophones d'autres régions, notamment de Toronto et d'Ottawa, se sont montrés intéressés à explorer des initiatives possibles. Une rencontre, le 3 décembre dernier, avec le responsable de Villes et villages en santé au Québec, a incité certains groupes du nord de l'Ontario (Dubreuilleville, Kapuskasing, Timmins, Chapleau, Noëlville) à passer à l'action. Notons qu'il pourrait y avoir plusieurs initiatives dans une même région ou encore dans une même communauté d'intérêt dans le but de répondre aux besoins spécifiques d'une communauté.

\section{Conclusion}

Ce qui n'était au départ qu'un concept plus ou moins abstrait prend maintenant de plus en plus l'allure d'un véritable mouvement de fond, s'appuyant sur des bases solides dans différents secteurs, dont les municipalités et les établissements de santé et de services sociaux, auxquels s'ajoutent, de plus en plus, d'autres secteurs tels l'environnement, l'éducation, la culture, les loisirs, la sécurité publique, etc.

Les premiers résultats des projets locaux des communautés en santé nous encouragent à poursuivre et à accroître les efforts pour 
Reflets

soutenir ces initiatives de coopération locale. Nous ne doutons aucunement des capacités de créativité auxquelles la société ontarienne aura recours dans l'amélioration de la prise en charge de sa santé et de celle de ses citoyennes et citoyens.

\section{Bibliographie}

COMITÉ CONSULTATIF DE LA RECHERCHE EN SANTÉ (1986). Stratégie de la recherche en santé pour tous en l'an 2000: rapport d'un sous-comité du CCRS, Organisation mondiale de la santé. MINISTÈRE DE LA SANTÉ ET DU BIEN-ÊTRE SOCIAL (1974). Nouvelle perspective de la santé des Canadiens, Ottawa, Information Canada.

POIRIER, Louis (1991). Santé, Société, vol. 13, no 3-4, automne, pp.47-48. 